\title{
Pre-Extensively Drug-Resistant Mycobacterium tuberculosis
}

National Cancer Institute

\section{Source}

National Cancer Institute. Pre-Extensively Drug-Resistant Mycobacterium tuberculosis. NCI Thesaurus. Code C128432.

A strain of Mycobacterium tuberculosis that is characterized by resistance to isoniazid, rifampin, and one of the following: a) any of the fluoroquinolone antibiotics or b) one or more of three injectable drugs (kanamycin, capreomycin, amikacin). 